Pacific Journal of Mathematics

UNIQUENESS FOR CERTAIN SURFACES OF PRESCRIBED 


\title{
UNIQUENESS FOR CERTAIN SURFACES OF PRESCRIBED MEAN CURVATURE
}

\author{
Thomas I. VogeL
}

\begin{abstract}
The classical problem of liquid in a capillary tube concerns finding the minimum of a certain energy function, which leads to a surface of prescribed mean curvature. This paper weakens the hypotheses by considering local minima of the functional. It is shown that no new surfaces result.
\end{abstract}

1. Introduction. Although the theorems in this paper have fairly general hypotheses, they are motivated by the theory of capillary surfaces. The classical capillary problem is to study the shape of the surface formed by capillary action when a tube of general cross-section $\Omega \subseteq \mathbf{R}^{n}$ is placed into an infinite reservoir. The assumption that has customarily been made is that the surface is energy minimizing over compact perturbations. It is natural to ask whether a surface can exist which is a local minimum for the functional but not a global minimum. In other words, is it possible, in the classical problem, for an "exotic" capillary surface to exist which is stable with respect to small compact perturbations, but unstable with respect to a large compact perturbation? The paper proves that under fairly weak assumptions, the answer is "no".

Certainly there are other problems in capillarity with non-trivial local minima. The energy functional for the pendent drop (e.g. Wente [10]) has no minimum if arbitrarily large perturbations are allowed, since by falling to $-\infty$ a drop looses an infinite amount of potential energy. Another such situation is that of a drop trapped between two planes (Vogel [8]). For some values of the separation, the (stable) connected drop bridging the planes has a larger energy than a spherical cap on one plane.

The reason the assumption that the surface is energy minimizing over all perturbations has been made in the classical problem is that a result of Miranda [5] then implies that the surface is a graph. The idea of the proof is that replacing the set $U$ occupied by the liquid by the set $U^{s}=\left\{(x, t): t \leq \int_{0}^{\infty} \phi_{U}(x, \tau) d \tau\right\}$ will reduce perimeter and hence the relevant energy functional. Here $t=0$ is chosen to lie below the 
free surface, $x \in \mathbf{R}^{n}$, and $\phi_{U}$ is the characteristic function of $U$. If we restrict our attention to "small" compact perturbations, we no longer have this result, since the perturbation from $U$ to $U^{S}$ may be large.

A notational pitfall must be avoided here. The term "local minimum" is often used in BV theory (the theory of functions of bounded variation) to describe a set $U$ such that $\mathscr{E}(U) \leq \mathscr{E}(V)$ for all sets $V$ with $U \Delta V(=(U-V) \cup(V-U))$ a precompact set. In the calculus of variations, a local minimum is a minimum over perturbations which are sufficiently small in some sense. For example, the perturbation $U \rightarrow U^{s}$ referred to before is a compact perturbation of the set if the free boundary is bounded, but is not necessarily a "small" perturbation. To avoid confusion we will restrict the term "local minimum" to the sense used in the calculus of variations, and for "local minimum" in the sense of BV theory we will use "minimum under compact perturbations".

Since $U \rightarrow U^{s}$ may be a large perturbation, in seeking surfaces which are local minima we may no longer restrict ourselves to surfaces which are graphs. Indeed, if a local minimum exists which is not a global minimum, we would expect it not to be a graph, since otherwise uniqueness results apply.

This paper approaches the problem from two different points of view. In $\S 2$ we will use BV theory to show that a local minimum is a graph, to which we can apply uniqueness theorems to conclude that it must be the classical solution. In $\S 3$ the problem is approached geometrically. We assume the surface satisfies the Euler-Lagrange equations for the energy functional, so that it might not be a local minimum, but just a stationary point. The conclusion is the same, however, that the surface will be a graph and hence the classical solution to the problem.

2. Local minima. We will deal with local minima for the capillary problem in terms of BV theory. The standard reference for BV theory is Giusti [3], and its use in capillary problems is shown in Finn [2]. The regularity results in Giusti are stated for minimal surfaces, however they also apply to capillary surfaces. Assume that $\Omega$ is an open set in $\mathbf{R}^{n}$ with a piecewise differentiable boundary and let $Q=\Omega \times \mathbf{R}$ be the capillary tube. Define the energy functional $\mathscr{E}$ by

(1) $\mathscr{E}(V)=\int_{Q}\left|D \phi_{v}\right|+n \int_{Q} H(x, t) \phi_{v}(x, t) d x d t-\int_{\partial Q} \beta(s) \phi_{v} d s d t$.

In the second integral $x \in \mathbf{R}^{n}$ and $t \in \mathbf{R}$. $H(x, t)$ is a Lipschitz continuous function which is non-decreasing in $t$. (For the classical 
problem, $H(x, t)=\kappa t$, and $\beta(s)$ is the cosine of the contact angle). Following Finn [2], $\S 7.3$, we will minimize this in the following sense: for $T>0$ define

$$
\begin{aligned}
Q_{T} & =\Omega \times[-T, T], \\
\delta Q_{T} & =\partial \Omega \times[-T, T],
\end{aligned}
$$

and

(2) $\mathscr{E}_{T}(V)=\int_{Q_{T}}\left|D \phi_{v}\right|+n \int_{Q_{T}} H(x, t) \phi_{V}(x, t) d x d t-\int_{\delta Q_{T}} \beta \phi_{V} d s d t$.

Then $U$ is a minimum for $\mathscr{E}$ under compact perturbations if for all $T>0, \mathscr{E}_{T}(U) \leq \mathscr{E}_{T}(V)$ for any $V$ with $U \Delta V \subseteq Q_{T}$.

We will want to restrict our attentions to perturbations which are not only contained in some compact set but are also "small". Thus $U$ is a local minimum for $\mathscr{E}$ under compact perturbations if there exists an $\varepsilon^{*}>0$ independent of $T$ such that $\mathscr{E}_{T}(U) \leq \mathscr{E}_{T}(V)$ for all $V$ with $U \triangle V \subseteq Q_{T}$ and $\partial V$ within $\varepsilon^{*}$ of $\partial U$.

Given a Borel set $E \subseteq \mathbf{R}^{n+1}$, we may change it by a set of measure zero so that

$$
0<|E \cap B(y, \rho)|<\omega_{n+1} \rho^{n+1}
$$

for all $y \in \partial E$ and $\rho>0$, where $B(y, \rho)$ is the ball of radius $\rho$ centered at $y$ and $\omega_{n+1}$ is the measure of the unit ball in $\mathbf{R}^{n+1}$ (Giusti [3], Proposition 3.1). We will assume that all sets we deal with in this paper have been so normalized.

1. LeMMA. Suppose $U, V$ are open set in $\mathbf{R}^{n+1}$ with $H_{n+1}(\partial U)=0$, and that $V \subseteq \bar{U}$. Then $V \subseteq U$.

Proof. Consider a point $q \in \partial U$. By our normalization, for any $\rho>0$,

$$
0<\left|U^{c} \cap B(q, \rho)\right|
$$

(Here $U^{c}$ is the complement of $U$.) Since $H_{n+1}(\partial U)=0$, there must be points in $(\bar{U})^{c}$ contained in $B(q, \rho)$. Therefore $q \notin V$.

2. Theorem. Assume that $U$ is an open set which is a local minimum for $\mathscr{E}$ under compact perturbations, that $H(x, t)$ is a Lipschitz continuous function which is non-decreasing in $t$, and that $U$ satisfies:

I. $(\partial U) \cap Q$ is connected.

II. There exists an $\varepsilon_{0}>0$ such that for all $\varepsilon \in\left(0, \varepsilon_{0}\right),(U-(0, \varepsilon))-U$ is uniformly contained in a compact set $K$. Here $U-(0, \varepsilon)$ is the translation of $U$ downward by $\varepsilon$ units. 
III. For some $\left(x_{0}, t_{0}\right) \in \partial U$ and some $\varepsilon_{1}>0,\left(x_{0}, t\right) \in U$ for $t_{0}-\varepsilon_{1}<$ $t<t_{0}$. We may assume that $\varepsilon_{1} \leq \min \left(\varepsilon_{0}, \varepsilon^{*} / 2,1\right)$.

Then there exists a function $u(x): \Omega \rightarrow[-\infty, \infty]$ such that $U=$ $\{(x, t): t<u(x)\}$.

Proof. We will denote $U-(0, \varepsilon)$ by $U_{\varepsilon}$. For any $\varepsilon$ and $T>0, U_{\varepsilon}$ is a local minimum under compact perturbations for:

$$
\begin{aligned}
\mathscr{E}_{\varepsilon}(V)= & \int_{Q_{T}}\left|D \phi_{V}\right|+n \int_{Q_{T}} H(x, t+\varepsilon) \phi_{V}(x, t) d x d t \\
& -\int_{\delta Q_{T}} \beta(s) \phi_{V}(s, t) d s d t
\end{aligned}
$$

Choose $T$ large enough so that $K \subseteq \Omega \times[-T+1, T-1]$. For any $\varepsilon \in\left(0, \varepsilon_{1}\right)$, we have that outside of $Q_{T}, U_{\varepsilon} \subseteq U$, so that

$$
\mathscr{E}_{\varepsilon}\left(U_{\varepsilon}\right) \leq \mathscr{E}_{\varepsilon}\left(U_{\varepsilon} \cap U\right)
$$

and

$$
\mathscr{E}_{T}(U) \leq \mathscr{E}_{T}\left(U_{\varepsilon} \cup U\right),
$$

since the free boundary of $U_{\varepsilon}$ is within $\varepsilon^{*} / 2$ of that of $U$. By an argument essentially the same as the proof of Theorem 5 of Vogel [7],

$$
\mathscr{E}_{\varepsilon}\left(U_{\varepsilon} \cap U\right)+\mathscr{E}_{T}\left(U_{\varepsilon} \cup U\right) \leq \mathscr{E}_{\varepsilon}\left(U_{\varepsilon}\right)+\mathscr{E}_{T}(U) .
$$

It follows that (3) and (4) are equalities. From this we see that $U_{\varepsilon} \cap$ $U$ is a local minimum for $\mathscr{E}_{\varepsilon}$ under compact perturbations, since a perturbation of $U_{\varepsilon} \cap U$ which moves the free boundary by less than $\varepsilon_{1}$ will have its free boundary within $\varepsilon^{*}$ of that $U_{\varepsilon}$. Similarly $U_{\varepsilon} \cup U$ is a local minimum under compact perturbations for $\mathscr{E}_{T}$.

We will now see that if $\left(x_{1}, t_{1}\right) \in U$, then $\left(x_{1}, t\right) \in U$ for $t \in\left(t_{1}-\right.$ $\left.\varepsilon_{1}, t_{1}\right]$. In other words, we want to show that $U_{\varepsilon} \subseteq U$ for all $\varepsilon \in\left[0, \varepsilon_{1}\right]$. By assumption III, $\left(\partial U_{\varepsilon}\right) \cap U$ is non-empty for $\varepsilon \in\left[0, \varepsilon_{1}\right]$. Suppose that there is a point $q \in Q-U$ which is contained in $U_{\varepsilon}$ for some positive $\varepsilon$ less that $\varepsilon_{1}$. If $H_{n+1}(\partial U)=0$, we may assume, by Lemma 1 , that $q \in Q-\bar{U}$. However, since $U$ is a local minimum for $\mathscr{E}$ under compact perturbations, we may apply regularity results. The reduced boundary of $U$ has dimension $n$, and the singular part of $\partial U$ has dimension $n-7$, so that, indeed, $H_{n+1}(\partial U)=0$. Let $\varepsilon_{2}>0$ be the infimum of the set of $\varepsilon$ 's such that $q \in U_{\varepsilon}$. Clearly $q \in \partial U_{\varepsilon_{2}}$.

We may decompose $\left(\partial U_{\varepsilon_{2}}\right) \cap Q$ into three parts: $\left(\partial U_{\varepsilon_{2}}\right) \cap U,\left(\partial U_{\varepsilon_{2}}\right) \cap$ $(Q-\bar{U})$, and a third set which we will call $A . A$ is contained in 
$\left(\partial U_{\varepsilon_{2}}\right) \cap \partial U$, but it only includes points where the two boundaries actually cross. Since $U$ and $Q-\bar{U}$ are both open and $\left(\partial U_{\varepsilon_{2}}\right) \cap U$ and $\left(\partial U_{\varepsilon_{2}}\right) \cap(Q-\bar{U})$ are non-trivial, both of the latter two sets have Hausdorff dimension $n$. We wish to conclude that $A$ has Hausdorff dimension of at least $n-1$, despite possible singularities on $\partial U_{\varepsilon_{2}}$. A similar result is given by Bombieri [1] in the proof of the Strong Sobolev inequality for minimal hypersurfaces. Since the argument depends on blow-up sequences, it will work for the surfaces considered in the present paper as well.

The singular sets for $\partial U_{\varepsilon_{2}} \cap Q, \partial U \cap Q$, and $\partial\left(U \cup U_{\varepsilon_{2}}\right)$ have Hausdorff dimension of at most $n-7$. Since $A$ is in each of these hypersurfaces, there is a point $w \in A$ which is regular for all three hypersurfaces. There then exists a ball $B$ centered at $w$ such that all three surfaces are regular inside $B$, and moreover

$$
H_{n}\left(\partial U_{\varepsilon_{2}} \cap \partial\left(U \cup U_{\varepsilon_{2}}\right) \cap B\right)>0
$$

and

$$
H_{n}\left(\partial U \cap\left(\partial U \cup U_{\varepsilon_{2}}\right) \cap B\right)>0 .
$$

These last two inequalities hold since $\partial U$ and $\partial U_{\varepsilon_{2}}$ actually cross at $w$.

Both $\partial U$ and $\partial\left(U \cup U_{\varepsilon_{2}}\right)$ may be expressed in $B$ as graphs of $C^{2}$ solutions $v_{1}$ and $v_{2}$ of the elliptic differential equation of prescribed mean curvature

$$
\operatorname{div} T v=\tilde{H}(\tilde{x}, v),
$$

where the tildes are due to the fact that the coordinate system may be rotated. Even so, $\tilde{H}$ will be Lipschitz continuous since $H$ is. However, since the graphs of $v_{1}$ and $v_{2}$ coincide on a set of Hausdorff dimension $n$, the strong unique continuation property (see Miranda [4]) implies that the graphs of $v_{1}$ and $v_{2}$ are identical in $B$. This contradicts $\partial U$ and $\partial U_{\varepsilon_{2}}$ crossing at $w$, which came from assuming that $U_{\varepsilon}-U$ was non-empty for some $\varepsilon \in\left[0, \varepsilon_{1}\right]$. Thus $U_{\varepsilon} \subseteq U$ for all $\varepsilon \in\left[0, \varepsilon_{1}\right]$.

It now follows that $U_{\varepsilon} \subseteq U$ for any $\varepsilon>0$. Indeed, given $\varepsilon>0$ we find $n$ so that $\varepsilon / n<\varepsilon_{1}$. Then

$$
U \supseteq U_{\varepsilon / n} \supseteq U_{2 \varepsilon / n} \supseteq \cdots \supseteq U_{\varepsilon}
$$

by a simple induction. We may therefore define $u(x)$ by

$$
u(x)=\sup _{t}\{(x, t):(x, t) \in U\} \text {. }
$$

$U$ will be the subgraph of $u(x)$, since for fixed $x,\{(x, t):(x, t) \in U\}$ is a half-open interval extending to $-\infty$. 
3. REMARK. Two conditions that will imply conditions II and III are: $T_{2}$.

IV. $\Omega \times\left(-\infty, T_{1}\right] \subseteq U \subseteq \Omega \times\left(-\infty, T_{2}\right]$ for some numbers $T_{1}$ and

Or more generally,

V. $\left(\Omega \times\left(-\infty, T_{1}\right]\right) \cap U$ is the subgraph of some function defined on $\Omega$, and so is $\left(\Omega \times\left(-\infty, T_{2}\right]\right) \cup U$, for some numbers $T_{1}$ and $T_{2}$.

Essentially, condition IV is saying that $\partial U \cap Q$ is bounded, and condition $\mathrm{V}$ requires that outside of a compact set, $\partial U \cap Q$ is a graph over $\Omega$.

4. Remark. Condition III will be true if $\partial U$ lies above $U$ in any neighborhood. This will certainly occur if the outward pointing normal at some regular point of $\partial U$ has a positive $t$ component. Some condition such as this is necessary, since for $H \equiv 0, \beta \equiv 0$, the set $U$ lying above a horizontal plane is a local minimum under compact perturbations. This is a supergraph, not a subgraph.

5. REMARK. The method of Theorem 2 also may be used to show that two sets $U$ and $V$ which are local minima under compact perturbations and satisfy condition IV cannot have free boundaries that cross. The method of proof is to lower $V$ until it is just contained in $U$, and then raise it by $\varepsilon^{*} / 2$. A contradiction is arrived at by looking at $U \cup V$ and $U \cap V$ as before.

6. Note. Thus far, we have not needed the assumption that $\partial \Omega$ is piecewise differentiable. We only needed enough regularity to make the last integral in equation (1) meaningful.

At this point, however, we want to show that a local minimum under compact perturbations is the same as the variational solution (see Finn [2], Chapter 7). Obviously we will need to impose enough conditions on the problem to ensure the existence of a variational solution.

7. Lemma. Let $U=\{(x, t) \mid t<u(x)\}$ be a local minimum for $\mathscr{E}$ under compact perturbations. If $\lim _{t \rightarrow \infty} H(x, t)=\infty$ uniformly in $x$, then the set $P=\{x \mid u(x)=\infty\}$ has measure zero. If $\lim _{t \rightarrow-\infty} H(x, t)=$ $-\infty$ uniformly in $x$, then $N=\{x \mid u(x)=-\infty\}$ has measure zero.

Proof. The argument is essentially the same as that of Miranda for minima over compact perturbations (see Finn [2], §7.5). I claim that $P$ is a local minimum of

$$
\Phi\left[\Omega^{*}\right]=|\Gamma|-\int_{\Sigma^{*}} \beta d s+n H_{\infty}\left|\Omega^{*}\right|
$$


where $\Gamma=\partial \Omega^{*}-\partial \Omega, \Sigma^{*}=\partial \Omega^{*} \cap \partial \Omega$, and $H_{\infty}=\lim _{t \rightarrow \infty} H(x, t)$. Once this is established, the proof is done, since $H_{\infty}=\infty$.

We now prove the claim. The translations $U-(0, M)$ approach $P \times R$ uniformly as $M$ goes to $\infty$. Suppose that $P$ is not a local minimum of $\Phi$. Fix $T>0$. For $M$ sufficiently large, a perturbation $\tilde{P}$ of $P$ which will reduce $\Phi$ may be used construct a perturbation $\tilde{U}$ of $U-(0, M)$ with $\tilde{U} \Delta(U-(0, M)) \subseteq Q_{T}$ and which has a smaller energy. The details are essentially the same as the proof of Theorem 13 of Vogel [9] and are omitted. The proof that $N$ has measure zero uses upward translations but is otherwise identical.

Now we use the argument which proves Proposition 16.7 in Giusti [3] to conclude that $u$ is locally bounded. In adapting this proof the assumption that $\varepsilon^{*}$ is independent of $T$ in the definition of a local minimum is used. It now follows, as in Giusti [3], that the function $u(x)$ for which $U$ is a subgraph is real analytic in $\Omega$ and satisfies

$$
\operatorname{div}\left(\frac{\nabla u}{\sqrt{1+|\nabla u|^{2}}}\right)=H(x, u)
$$

there.

8. THEOREM. Let each point $p \in \partial \Omega$ satisfy the hypotheses of Theorem 7.5 in Finn [2]. Then there is only one set $U$ which satisfies the hypotheses of Theorem 1 and it is in fact a minimum over compact perturbations.

Proof. From Lemma 7, $U$ is the subgraph of a function $u: \Omega \rightarrow$ $(-\infty, \infty)$ satisfying $(5)$. We must verify that $u$ is a variational solution in the sense of Definition 7.1 of Finn [2]. What that involves is showing that the arguments in $\S 7.5$ of Finn [2] still work for local minima, i.e. if we require perturbations to be small. But this is straight-forward to check.

Now that we know that $u$ is a variational solution, we may apply Finn's Theorem 7.6 for uniqueness. Of course a minimum for the functional over compact perturbations is known to exist.

3. Surfaces of prescribed mean curvature, without assumptions on the energy functional. We will now consider a somewhat different situation. For the open set $U$, assume that $\partial U \cap Q$ is a smooth surface $S$ whose mean curvature at a point $(x, t) \in S$ is $H(x, t)$, with $H(x, t)$ either constant or a Lipschitz continuous function which is strictly increasing in $t$. We are not assuming that $U$ is even a local minimum 
for $\mathscr{E}$. By the regularity results for capillary surfaces [3], for $n<7$ this is a weaker assumption on the free surface than that of the previous section. The weaker assumption on the free surface is balanced by stronger assumptions on the boundary conditions.

9. THEOREM. Suppose that $\partial \Omega$ is differentiable and that $U \subseteq Q$ satisfies the condition that $U_{t_{1}} \subseteq U$ for some $t_{1}>0$, where $U_{t}=U-(0, t)$ is the downward translation of $U$ by $t$ units. We make the following assumptions on $S=\partial U \cap Q$ :

(i) The curvature of $S$ at $(x, t)$ is $H(x, t)$, where either $H(x, t)$ is strictly increasing in $t$ or $H$ is constant.

(ii) $S$ is a bounded set, and if $H$ is constant, $S$ is assumed to be connected.

(iii) The contact curve $\bar{S} \cap \partial Q$ is $C^{2}$, and $S$ may be continued differentiably for a small distance outside of $Q$.

(iv) The angle between the outer normal to $S$ and the outer normal to $\partial Q$ at a point $(s, t)$ is $\gamma(s)$.

Then there is a function $u(x): \Omega \rightarrow(-\infty, \infty)$ such that $U=\{(x, t)$ : $t<u(x)\}$.

Proof. I first claim that for some $T, U \subseteq(-\infty, T] \times \Omega$. Suppose that this is not the case. Let $T_{1}$ be the supremum of the $t$ coordinates of points in $S$. Since $U$ is assumed not to be contained in $\left(-\infty, T_{1}\right] \times \Omega$, there must be points of $U$ lying above the plane $t=T_{1}$. But since $Q$ contains no boundary points of $S$ above $t=T_{1}$, it follows that $\left(T_{1}, \infty\right) \times \Omega \subseteq U$. We now use the assumption that $U_{t_{1}} \subseteq U$ to conclude that $\left(T_{1}-t_{1}, \infty\right) \times \Omega \subseteq U$. But then there can be no points of $S$ above $t=T_{1}-t_{1}$, a contradiction. Hence $U \subseteq\left(-\infty, T_{1}\right] \times \Omega$.

Let $W=\left\{t \mid U_{t} \subseteq U\right\}$. Observe that $W \subseteq[0, \infty)$. Indeed, if $U_{t} \subseteq U$ for a negative value of $t$, this corresponds to an upward translation of $U$. But this will take points of $U$ near $t=T_{1}$ above that plane, a contradiction. Now, let $W_{0}$ be the connected component of $W$ containing $t_{1}$, so that $W_{0}$ is a point or a line segment, and let $t_{0}=\inf W_{0}$. We will see that $t_{0}$ is zero. The first observation is that $t_{0} \in W$ and hence in $W_{0}$. Indeed, if this were not the case, there would be a ball of radius $\rho>0$ contained in $U_{t_{0}}-U$, so that $U_{t_{0}+\delta}$ would also not be contained in $U$ for $\delta \in[0, \rho)$. This contradicts the choice of $t_{0}$. We also have $\overline{U_{t_{0}}} \nsubseteq U$, since if $\overline{S_{t_{0}}}$ and $\bar{S}$ do not intersect, the distance between these two sets is positive. We obtain a contradiction as before. This argument uses the boundedness of the free surface.

Now let $p=\left(x^{*}, t^{*}\right) \in \bar{S} \cap \bar{S}_{t_{0}}$. There are two cases to consider. 
Case 1. $p \in Q$. Then $p \in S \cap S_{t_{0}}$. The mean curvature of $S_{t_{0}}$ at $p$ is $H\left(x^{*}, t^{*}+t_{0}\right)$, and that of $S$ is $H\left(x^{*}, t^{*}\right)$. We must verify that the outward pointing normals to $U$ and $U_{t_{0}}$ coincide at $p$. That $S$ and $S_{t_{0}}$ are tangent at $p$ is clear, hence the normals must point in the same or opposite directions. but since $U_{t_{0}} \subseteq U$, the former must hold. Call this normal vector $\vec{n}$.

If $H$ is strictly increasing in $t$, we obtain a contradiction through an elementary argument. Consider $n$ perpendicular planes $\Pi_{1}, \Pi_{2}, \ldots, \Pi_{n}$ through $p$ containing $\vec{n}$. Let $C^{i}, C_{t_{0}}^{i}$ be the intersections of $S$ and $S_{t_{0}}$ with $\Pi_{i}, i=1, \ldots, n$. The curvatures of $C^{i}$ at $p$ with respect to $\vec{n}$ must sum to $n H\left(x^{*}, t^{*}\right)$ and those of $C_{t_{0}}^{i}$ sum to $n H\left(x^{*}, t^{*}+t_{0}\right)$ (it is not necessary to assume that these are the principal curvatures. See Finn [2], §1.1). If $t_{0}>0$, this will mean that the curvature of $C_{t_{0}}^{i}$ is larger than that of $C^{i}$ for some $i$. This contradicts the fact that $U_{t_{0}} \subseteq U$.

If $H$ is constant, then the contradiction comes from the touching principle resulting from the Hopf maximum principle (see Wente [10], $\S 2)$.

Case 2. $p \in \partial Q$. We must again prove that the outer normals coincide at $p$. Then tangent spaces to $\bar{S}$ and $\bar{S}_{t_{0}}$ at $p$ cannot cross in $Q$, thus the projections of $\vec{n}$ and $\vec{n}_{t_{0}}$ into the tangent space to $\partial Q$ at $p$ must be parallel. In fact, they must be equal, since the trace of $U_{t_{0}}$ on $\partial Q$ is contained in the trace of $U$. Pick a coordinate system of $\mathbf{R}^{n+1}$ at $p$ with $\vec{\nu}$, the normal to $\partial Q$ at $p$, as one of the coordinate vectors. The other coordinates of $\vec{n}$ and $\vec{n}_{t_{0}}$ are already known to be equal. The components of $\vec{n}$ and $\vec{n}_{t_{0}}$ along $\vec{\nu}$ are equal by the contact angle condition, thus $\vec{n}$ and $\vec{n}_{t_{0}}$ coincide.

Now pick $n$ orthogonal unit vectors $\vec{v}_{1}, \ldots, \vec{v}_{n}$ in the tangent space to $\bar{S}$ (and $\bar{S}_{t_{0}}$ ) at $p$. The argument proceeds as in Case 1 , letting $\Pi^{i}$ contain $\vec{n}$ and $\vec{v}_{1}$. We obtain curves $C^{i}$ which end at $p$, but the same contradiction is reached. Hence $t_{0}=0$.

Now let $t_{2}=\sup \left(W_{0}\right)$. If $t_{2}$ is finite we obtain a contradiction as before. Therefore $W=W_{0}=[0, \infty)$. The theorem now follows, since if $\left(x_{0}, a\right) \notin U$ and $\left(x_{0}, b\right) \in U$ with $a<b$, it would follow that $b-a \notin W$. For fixed $x,\left\{t \mid\left(x_{0}, t\right) \in U\right\}$ is an interval extending to $-\infty$, and we may therefore define $u(x)=\sup \{t \mid(x, t) \in U\}$. 
10. Note. Although we have shown that $S$ is the graph of $u(x)$, it does not immediately follow that $u(x)$ is a classical solution to

$$
\begin{aligned}
& \operatorname{div}\left(\frac{\nabla u}{\sqrt{1+|\nabla u|^{2}}}\right)=H(x, u) \text { in } \Omega, \\
& \vec{N} \cdot\left(\frac{\nabla u}{\sqrt{1+|\nabla u|^{2}}}\right)=\cos \gamma(s) \text { on } \partial \Omega .
\end{aligned}
$$

where $\vec{N}$ is the outer normal. The problem is that at this point we do not know whether $\nabla u$ is bounded in $\Omega$, i.e. whether $S$ can become vertical. If, however, we assume that $H(x, t)$ is a $C^{1}$ function, the argument in the proof of Theorem 14.10 of Giusti [3] may be adapted to eliminate vertical points.

11. REMARK. We may replace hypotheses (iii) and (iv) of Theorem 9 by the assumption that the contact curve $\bar{S} \cap \partial Q$ is a graph. Then Case 2 of Theorem 9 cannot occur, so that no other boundary conditions are needed. For $H=$ constant, this was proven by Serrin [6].

12. REMARK. The ideas in this section may be used to strengthen a theorem of the author's on asymmetric liquid bridges (Theorem 3.7 of [7]). The theorem is that if a flat object $F$ satisfying an internal sphere condition of radius $R$ is withdrawn a sufficiently small distance $h<h(R)$ from an infinite pool of liquid, the bridge surface formed will be a graph. In the paper cited, however, the additional assumption that the bridge surface is known to be a graph sufficiently far from $F$ is made. This may be replaced by the more natural assumption that the height of the bridge surface approaches zero at infinity.

The proof follows the same lines as that of Theorem 9. From [7], Theorem 3.4, the point of first contact between $\bar{S}$ and $\bar{S}_{t_{0}}$ cannot be made at the boundary of either surface, and by the assumption on the free surface, the first contact cannot occur at infinity. Thus, we may argue as in Case 1 of Theorem 9 to obtain a contradiction.

13. REMARK. The conditions on the contact angle may be weakened in both $\S \S 2$ and 3 with no substantive change in the proofs. In $\S 2, \beta(s)$ may be replaced by $\beta(s, t)$, a non-increasing function of $t$. In $\S 3, \gamma(s)$ may be similarly replaced by $\gamma(s, t)$, a non-decreasing function of $t$.

I would like to thank Robert Finn for his continuing interest in this work, and also the Department of Mathematics of the Ohio State University for the hospitality shown during my visit there. I would 
also like to thank Jon Pitts for several useful conversations, and Enrico Giusti for suggesting a substantial improvement to Theorem 2 .

\section{REFERENCES}

[1] E. Bombieri, An Introduction to Minimal Currents and Parametric Variational Problems, Mathematical Reports, vol. 2, (1985), 285-384, Harwood Academic Publishers, London.

[2] R. Finn, Equilibrium Capillary Surfaces, Springer-Verlag, New York, Berlin, 1986.

[3] E. Giusti, Minimal Surfaces and Functions of Bounded Variation, Birkhauser, Boston, 1984.

[4] C. Miranda, Partial Differential Equations of Elliptic Type, 2nd revised ed., trans. by Z. C. Motteler, Springer-Verlag, New York, Berlin, 1970.

[5] M. Miranda, Superfici cartesiane generalizzate ed insiemi di perimetro localmente finito sui prodotti cartesiani, Ann. Scuola Norm. Sup.-Pisa, n. 3 (1976), 501-548.

[6] J. Serrin, On surfaces of constant mean curvature which span a given space curve, Math. Z., 112 (1969), 77-88.

[7] T. I. Vogel, Asymmetric unbounded liquid bridges, Ann. Scuola Norm. Sup.-Pisa, Cl. di Sci, ser. 4, vol. 9, no. 3 (1982), 433-442.

[8] Stability of a liquid drop trapped between two parallel planes, SIAM J. Appl. Math., 47, no. 3 (1987), 516-525.

[9] Unbounded parametric surfaces of prescribed mean curvature, Indiana Univ. Math. J., 31, no. 2 (1982), 281-288.

[10] H. C. Wente, The stability of the axially symmetric pendent drop, Pacific J. Math., 88, no. 2 (1980), 421-470.

[11] - The symmetry of sessile and pendent drops, Pacific J. Math., 88, no. 2 (1980), 387-397.

Received March 5, 1987.

TEXAS A \& M University

College Station, TX 77843 



\section{PACIFIC JOURNAL OF MATHEMATICS EDITORS}

V. S. VARADARAJAN (Managing Editor) University of California Los Angeles, CA 90024

HeRbert Clemens

University of Utah

Salt Lake City, UT 84112

R. FINN

Stanford University

Stanford, CA 94305
ROBION KIRBY

University of California

Berkeley, CA 94720

C. C. MOORE

University of California

Berkeley, CA 94720

HAROLD STARK

University of California, San Diego

La Jolla, CA 92093

\section{ASSOCIATE EDITORS}
R. ARENS
E. F. BECKENBACH
B. H. NEUMANN
F. Wolf
K. YOSHIDA (1906-1982)

\section{SUPPORTING INSTITUTIONS}

UNIVERSITY OF ARIZONA UNIVERSITY OF OREGON

UNIVERSITY OF BRITISH COLUMBIA UNIVERSITY OF SOUTHERN CALIFORNIA

CALIFORNIA INSTITUTE OF TECHNOLOGY UNIVERSITY OF CALIFORNIA MONTANA STATE UNIVERSITY STANFORD UNIVERSITY

UNIVERSITY OF NEVADA, RENO UNIVERSITY OF HAWAII NEW MEXICO STATE UNIVERSITY UNIVERSITY OF TOKYO UNIVERSITY OF UTAH OREGON STATE UNIVERSITY WASHINGTON STATE UNIVERSITY UNIVERSITY OF WASHINGTON 


\section{Pacific Journal of Mathematics}

Vol. 134, No. $1 \quad$ May, 1988

Marco Abate, Annular bundles . ............................

Ralph Cohen, Wen Hsiung Lin and Mark Mahowald, The Adams

spectral sequence of the real projective spaces

Harry Joseph D'Souza, Threefolds whose hyperplane sections are elliptic surfaces .........................................57

Theodore Gerard Faticoni, Localization in finite-dimensional FPF rings . . . 79

Daniel Hitt, Invariant subspaces of $\mathscr{H}^{2}$ of an annulus ................ 101

Ellen Kirkman and James J. Kuzmanovich, On the global dimension of fibre products

Angel Rafael Larotonda and Ignacio Zalduendo, Homogeneous spectral sets and local-global methods in Banach algebras

Halsey Lawrence Royden, Jr., Invariant subspaces of $\mathscr{H}^{p}$ for multiply

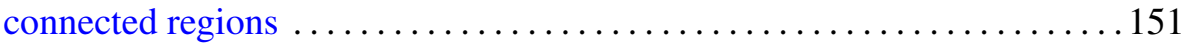

Jane Sangwine-Yager, A Bonnesen-style inradius inequality in 3-space . . . . 173

Stefano Trapani, Holomorphically convex compact sets and cohomology

Thomas Vogel, Uniqueness for certain surfaces of prescribed mean

curvature 